\title{
Fenología reproductiva de las especies arbóreas del bosque tropical de Tenosique, Tabasco, México
}

\author{
Susana Ochoa-Gaona, Isidro Pérez Hernández \& Bernardus H.J. de Jong \\ El Colegio de la Frontera Sur, Departamento de Agroecología, Apdo. Postal 1042, Admón. de Correos de Tabasco \\ 2000. C.P. 86031 Villahermosa, Tabasco, México; sochoa@ecosur.mx; iperez@ecosur.mx; bjong@ecosur.mx
}

Recibido 14-XI-2006. Corregido 25-X-2007. Aceptado 05-XI-2007.

\begin{abstract}
Reproductive phenology of tree species in the Tenosique tropical forest, Tabasco, Mexico. Between August 2003 and August 2005 we registered the flowering and fruiting of 75 tree species (341 individual trees) in a tropical rain forest at Tenosique, Tabasco, Mexico. Monthly we checked five transects (500 $\mathrm{m}$ long; $5 \mathrm{~m}$ wide). To test the homogeneity of flowering and fruiting during the year, and between adjacent months, we applied a $\chi^{2}$ test. The flowering was bimodal, with a highest peak in March and April, coinciding with the dry season, and a second lower peak in July when precipitation is relatively low. The highest number of fruiting tree species occur between May and July, with its peak in May. Each of the most common botanical families showed a particular phenological pattern. Monthly rainfall and the number of species flowering or fruiting were not significantly correlated. This means that trees are flowering and fruiting all year long, with seasonal increases of both phenological phenomena in the dryer periods. We conclude that phenological patterns vary between individuals and between years and are not seasonally correlated. The data we generated are relevant to program the best periods of seed collections according to individual or groups of species, as part of forest management and conservation practices. Rev. Biol. Trop. 56 (2): 657-673. Epub 2008 June 30.
\end{abstract}

Key words: phenophases, reproductive biology, tropical forest, tropical trees, woody flora.

La fenología es el estudio de las fases o actividades periódicas y repetitivas del ciclo de vida de las plantas y su variación temporal a lo largo del año (Mantovani et al. 2003, ICPForest 2006, Preuhsler et al. 2006). El conocimiento fenológico contribuye al entendimiento de los patrones reproductivos y vegetativos de las plantas y de los animales que de ellas dependen, entre ellos herbívoros, polinizadores, y frugívoros (Justiniano y Fredericksen 2000, Mantovani et al. 2003). La fenología puede contribuir a la solución de algunos problemas forestales, ya que sienta bases para comprender la biología de la reproducción de las especies, la dinámica de las comunidades, las interacciones planta-animal y la evolución de la historia de vida de los animales que dependen de las plantas para su alimentación (Talora y
Morellato 2000, Vílchez y Rocha 2004). Este conocimiento proporciona información sobre la disponibilidad de recursos a lo largo del año y permite determinar las estrategias de recolecta de frutos, lo que puede favorecer la calidad y cantidad de semillas para la producción de nuevas plántulas (Mantovani et al. 2003).

A través del estudio de la fenología, se tratan de establecer las posibles causas de su presencia con relación a factores bióticos y abióticos (Talora y Morellato 2000, Vílchez y Rocha 2004). Entre los factores abióticos que pueden influir en la variación temporal de la fenología reproductiva de las especies, se ha enfatizado en las horas de brillo solar, la humedad relativa, la temperatura y la precipitación, siendo ésta última, la principal variable estudiada en la fenología tropical (Vílchez y Rocha 
2004). En este sentido, Longino (1986), en un estudio realizado en el Parque Corcovado en Costa Rica, encontró una correlación negativa entre la cantidad de lluvia y la producción de brotes de Passiflora pittieri. Sun et al. (1996), en un estudio realizado en Rwanda con 49 especies arbóreas, mencionan que el pico de fructificación ocurrió durante la estación mas húmeda entre marzo y mayo, pero ésta, permaneció alta durante el periodo de sequía. Por su parte, Vílchez et al. (2004) en un estudio realizado en Costa Rica, en el que se evalúa la época reproductiva de cinco especies forestales del bosque secundario tropical, encontraron baja correlación entre la precipitación y las fenofases estudiadas.

El conocimiento de las épocas de floración y fructificación es importante para la conservación de recursos genéticos y el manejo forestal de bosques primarios, secundarios pues marca los meses en los que ocurre, ayudando a la planificación de colectas de semilla y la detección de las mejores procedencias de germoplasma (Plana 2000, Vílchez et al. 2004). El conocimiento de los sistemas de reproducción de las especies es muy importante en los trópicos, ya que en éstos, muchas especies presentan baja densidad natural, la cual se acentúa por actividades de extracción selectiva (Plana 2000). Por otra parte, se sabe que los ritmos anuales de los árboles responden a los cambios en el tiempo o condiciones climáticas, por ello, el estudio de la fenología es de interés especial por los efectos de calentamiento climático sobre la condición del bosque (Chuine y Beaubien 2001, ICP-Forest 2006).

A pesar de la gran importancia del conocimiento fenológico de las especies, éste aun es muy escaso y fragmentario particularmente en las regiones tropicales (Fournier y Charpantier 1975, Mantovani et al. 2003). Hasta la fecha, en México sólo las especies de mayor valor comercial han sido estudiadas detalladamente para su manejo, por ello resulta de particular importancia profundizar en el conocimiento de la fenología de la flora leñosa nativa y sus posibles relaciones con la precipitación. En este marco, el objetivo de este trabajo fue el de caracterizar la fenología reproductiva de las especies de árboles más comunes de los bosques de Tenosique, Tabasco para sentar bases para su manejo y conservación.

\section{MATERIALES Y MÉTODOS}

Área de estudio: el municipio de Tenosique se localiza en la cuenca del río Usumacinta, en la porción oriental del estado; se ubica entre $17^{\circ} 15^{\prime} 00^{\prime \prime}-17^{\circ} 40^{\prime} 48^{\prime \prime}$ N, y los 9059'09”91³8'16" W, con una extensión territorial de $1848 \mathrm{~km}^{2}$ (INEGI 2000). Limita al norte con el municipio de Balancán, al sur y al este con la República de Guatemala, y al oeste con el municipio de Emiliano Zapata y el estado de Chiapas.

En la parte sur del municipio - en sus límites con Guatemala - se localiza un pequeño macizo montañoso con altura máxima de 660 m (Gobierno del Estado 1997). Esta zona ocupa alrededor de 47000 ha que corresponden al $36 \%$ de la superficie montañosa del estado de Tabasco y es el área donde se encuentran los mayores remanentes de bosque tropical lluvioso del estado.

Presenta un clima cálido-húmedo en el que la temperatura media anual oscila entre 25.5 y $27{ }^{\circ} \mathrm{C}$. La precipitación alcanza los $3282 \mathrm{~mm}$ anuales con un máximo de $600 \mathrm{~mm}$ en septiembre y un periodo de menor precipitación de marzo a abril. Los suelos dominantes en esta área son del tipo Litosol de poca profundidad y limitados por un estrato rocoso dentro de los primeros diez centímetros. También se encuentran Acrisoles y Rendzinas, estos últimos, con alto contenido de materia orgánica y materiales calcáreos generalmente asociados a pendientes abruptas.

Esta zona montañosa, esta cubierta por fragmentos de bosque tropical lluvioso inmersos en un mosaico de vegetación secundaria de diferente edad derivada de la actividad agrícola y pastizales inducidos para la ganadería localizados en las partes planas u onduladas. Los remanentes de bosque tropical perennifolio - en su mejor estado - alcanzan los $30 \mathrm{~m}$ de altura 
y están conformados por tres estratos arbóreos y un estrato herbáceo conformado por aráceas, marantáceas y helechos, además se presentan lianas y varios tipos de orquídeas (Gobierno del Estado 1997). De las especies arbóreas mas comunes se pueden mencionar la caoba (Swietenia macrophylla), cedro (Cedrela odorata), macayo (Andira galeottiana), palma real (Sabal mexicana), corozo (Attalea butyracea), jobo (Spondias mombin), macuilís (Tabebuia rosea), ceiba (Ceiba pentandra), laurel (Nectandra ambigens), piche (Enterolobium cyclocarpum), árbol de hule (Castilla elastica), barí (Calophyllum brasiliense), y bojón (Cordia alliodora). Esta vegetación es de gran importancia a nivel estatal, por lo que se ha declarado área natural protegida con carácter de área de protección de flora y fauna (DOF 2005). Constituye uno de los últimos refugios de la fauna tropical del estado, en la que se tienen registradas 209 especies de aves, 132 de mamíferos, 29 de peces, 29 de reptiles y 20 de anfibios (Galindo et al. 2000). A pesar de esto, ha sido objeto de una tala intensiva a favor principalmente de la ganadería (Secretaría de Gobernación s/f).

Fenología: se establecieron cinco transectos fijos de $500 \mathrm{~m}$ de largo y $5 \mathrm{~m}$ de ancho cubriendo una superficie de $2500 \mathrm{~m}^{2}$ cada uno. Tres de estos transectos se localizaron en el ejido Nuevo Progreso y dos de ellos en el ejido Niños Héroes. Se registró un mínimo de cinco árboles por especie con un diámetro a la altura del pecho (dap) $\geq 10 \mathrm{~cm}$ para tratar de asegurar que los árboles ya estuvieran en su fase reproductiva (Fournier y Charpantier 1975). Los árboles seleccionados fueron marcados con etiquetas plásticas con pintura indeleble para facilitar la re-localización, asignando un número consecutivo (Preuhsler et al. 2006). Los transectos se evaluaron mensualmente (Justiniano y Fredericksen 2000, Talora y Morellato 2000) durante la primera semana de cada mes a partir de agosto del 2003 y hasta agosto del 2005.

La presencia de flores y frutos fue evaluada con la ayuda de binoculares. Si alguno de los individuos de la especie tenia flores o frutos, la especie como un todo fue considerada como en floración o fructificación en ese mes (Justiniano y Fredericksen 2000, Bencke y Morellato 2002).

Cada árbol fue censado, registrando su dap, su nombre común y la especie. Se realizó la recolecta botánica de cada especie, habiendo depositado los ejemplares en el herbario $\mathrm{CH}$ de ECOSUR-SCLC. La identificación se realizó mediante el uso de características dendrológicas comparando ejemplares con un catálogo de campo elaborado previamente por personal de Ecosur. Las especies nuevas se integraron a dicho catálogo. Las especies que no se lograron identificar por ésta vía, se identificaron mediante el uso de claves dendrológicas y taxonómicas (Steyermark 1950, Budowski 1954, Standley y Steyermark 1946-1960, Standley y Williams 1961-1975, Holdridge 1970, Gentry y Standley 1974, Nash y Williams 1976, Croat 1978, Cabrera et al. 1982, Ochoa-Gaona et al. 1990, Salas Estrada 1993, Pennington y Sarukhán 2005) y mediante comparación con material de los Herbarios de la UJAT y de Ecosur San Cristóbal (CH).

Se aplicó una prueba de homogeneidad de ji-cuadrada (Finney 1980, Zar 1999) para evaluar la hipótesis nula: a) que a lo largo del año el numero de especies leñosas que florecen es similar, b) que a lo largo del año el numero de especies leñosas que fructifican es similar, y c) que el patrón de floración de las especies es similar al de la fructificación.

Para estudiar la posible relación de las fases reproductivas con la precipitación, se recolectaron datos de la estación meteorológica de Boca del Cerro, Tenosique, Tabasco que es la más cercana al sitio de estudio. Se realizaron correlaciones de Spearman $\mathrm{p}<0.05$ (Zar 1999) entre el número de especies en cada fenofase, por mes y las variaciones de precipitación en el mismo período. Como porcentaje de precipitación se consideró, el promedio de la cantidad de lluvia del mes correspondiente con respecto al total de la precipitación anual. El porcentaje de fenofase se tomó como la cantidad de especies en floración/fructificación expresado en porcentaje mensual de especies en la fenofase 
correspondiente (Talora y Morellato 2000, Vílchez et al. 2004). Dado que no hubo diferencias significativas entre la temperatura mensual, no se aplicó la correlación de Spearman con esta variable.

\section{RESULTADOS}

Flora: se registraron un total de 341 individuos pertenecientes a 30 familias, 66 géneros y 75 especies. Las familias mejor representadas fueron: Rubiaceae (7 especies), Fabaceae (6), Meliaceae, Moraceae y Sapotaceae (5), y Anacardiaceae, Euphorbiaceae y Lauraceae (4 especies cada una). El $60 \%$ de las familias sólo presentó una o dos especies (Cuadro 1).

Floración: el número de especies que florece es significativamente diferente a lo largo del año $\left(\chi^{2}=38.47, \mathrm{p}<0.001, \mathrm{gl}=11\right)$. La floración fue bimodal con un periodo de mayor floración en marzo a abril (22 especies cada mes) que corresponden a menor precipitación y que de acuerdo con Sun y colaboradores (1996) es el periodo de mayor insolación. En julio se presentó el segundo pico de floración con 21 especies, correspondiendo igualmente a un decremento de la precipitación (Fig. 1). La prueba de $\chi^{2}$ mostró diferencias significativas en la floración entre el bimestre de enero-febrero y marzo-abril $\left(\chi^{2}=16.86, \mathrm{p}<0.001, \mathrm{gl}=1\right)$ y entre los bimestres julio-agosto con relación a septiembre-octubre $\left(\chi^{2}=4.57, \mathrm{p}<0.05, \mathrm{gl}=1\right.$; Fig. 1$)$.

Fructificación: el número de especies que fructifica varió significativamente a lo largo del año $\left(\chi^{2}=22.32, \mathrm{p}<0.05, \mathrm{gl}=11\right)$. En lo general se registró un porcentaje alto de especies fructificando a lo largo del año, teniendo mayor número de especies con fruto entre mayo y junio, sin embargo no se encontraron diferencias estadísticas de este bimestre con los bimestres cercanos. Por otra parte, el bimestre de septiembre-octubre, que corresponde al periodo de menor fructificación fue estadísticamente diferente a los bimestres anterior y posterior $\left(\chi^{2}=4.85, \mathrm{p}<0.05, \mathrm{gl}=1\right.$; Fig. 1$)$.

Fenología de las familias con mayor número de especies: las conductas fenológicas de las familias con mayor número de especies fueron distintas entre sí. Las Rubiaceae presentaron un periodo de floración durante marzo a mayo y un pico mas alto en agosto. Las Sapotaceae presentaron una floración trimodal con picos en mayo, julio y noviembre. La fructificación en las Rubiaceae mostró un mayor pico en abril, mientras que las Sapotaceae igualmente se manifiestan trimodales con picos en junio-julio, septiembre y noviembre-diciembre (Fig. 2). La familia Fabaceae mostró tres picos de floración en marzo-abril, en agosto y en noviembre-diciembre, la floración de las

CUADRO 1

Número de especies arbóreas por familia botánica

TABLE 1

Number of species per botanical family

Familia

Rubiaceae

Fabaceae

Meliaceae, Moraceae, Sapotaceae

Anacardiaceae, Euphorbiaceae, Lauraceae

Annonaceae, Apocynaceae, Sapindaceae, Tiliaceae

Bombacaceae, Boraginaceae, Burseraceae, Flacourtiaceae, Myrtaceae

Actinidiaceae, Aquifoliaceae, Araliaceae, Bignoniaceae, Cecropiaceae, Chrysobalanaceae, Clusiaceae, Combretaceae, Rutaceae, Simaroubaceae, Sterculiaceae, Ulmaceae, Verbenaceae

No. de Especies

7

6

5

4

3

2

1 

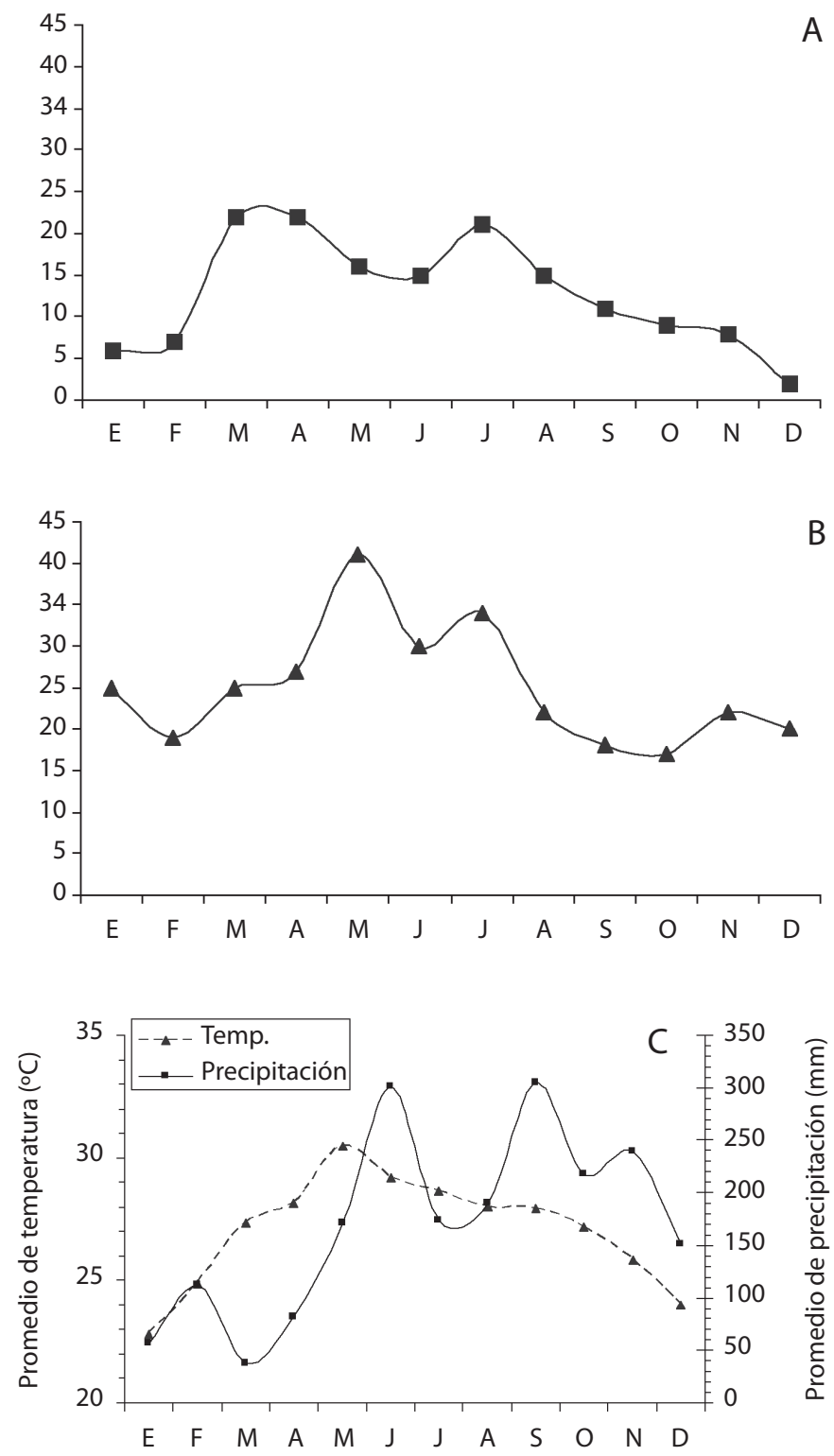

Fig. 1. Fenología de las especies arbóreas. (A) Número de especies en floración, (B) Número de especies en fructificación, y (C) Distribución del promedio de temperatura y precipitación de 2003-2005.

Fig. 1. Phenology of tree specie.s (A) Number of species in flowering period, (B) Number of species in fruiting period, (C) Mean of the temperature and precipitation for the period 2003-2005. 

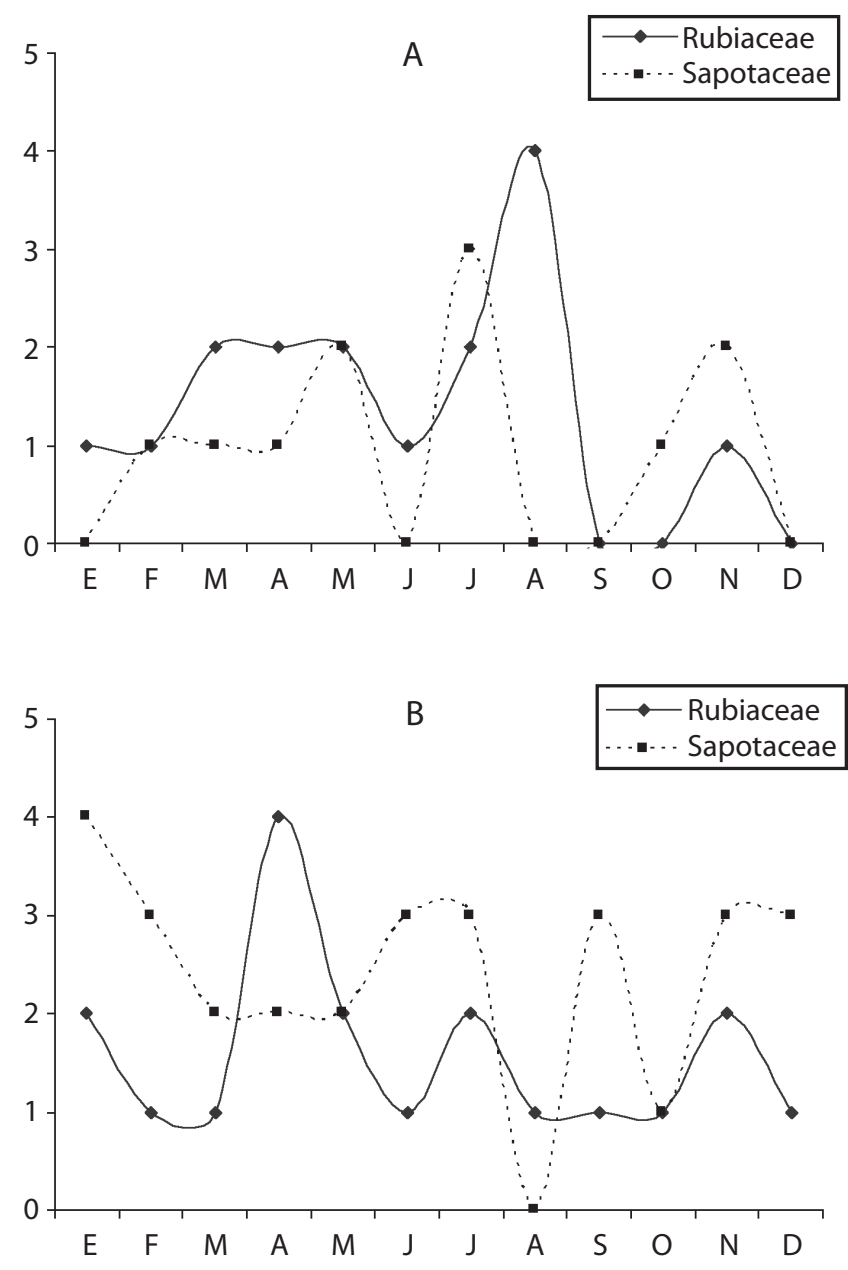

Fig. 2. Fenología de las familias Rubiaceae y Sapotaceae (A) Floración, (B) Fructificación.

Fig. 2. Phenology of Rubiaceae and Sapotaceae (A) Flowering, (B) Fruiting.

Meliaceae solo mostró un pico que se extiende de marzo a julio. Las Moraceae florecieron bimodalmente con picos en abril y julio. En cuanto a la fructificación, las Fabaceae y Moraceae se mostraron bimodales, con picos en mayo y julio, y en marzo y mayo respectivamente. Las Meliaceae mostraron un solo pico de fructificación en mayo (Fig. 3).

Relación entre precipitación y fenología: no se encontró ninguna relación significativa entre el número de especies floreciendo o fructificando con la cantidad de lluvia mensual.
Esta relación se evaluó también entre años $\mathrm{y}$ entre comunidades sin obtener resultados significativos.

\section{DISCUSIÓN}

Aunque no se encontró una relación significativa entre la fenología reproductiva y la precipitación, el pico de floración coincide en la época de menor precipitación, lo cual concuerda con lo reportado para especies arbóreas por Ochoa-Gaona y Domínguez-Vázquez 

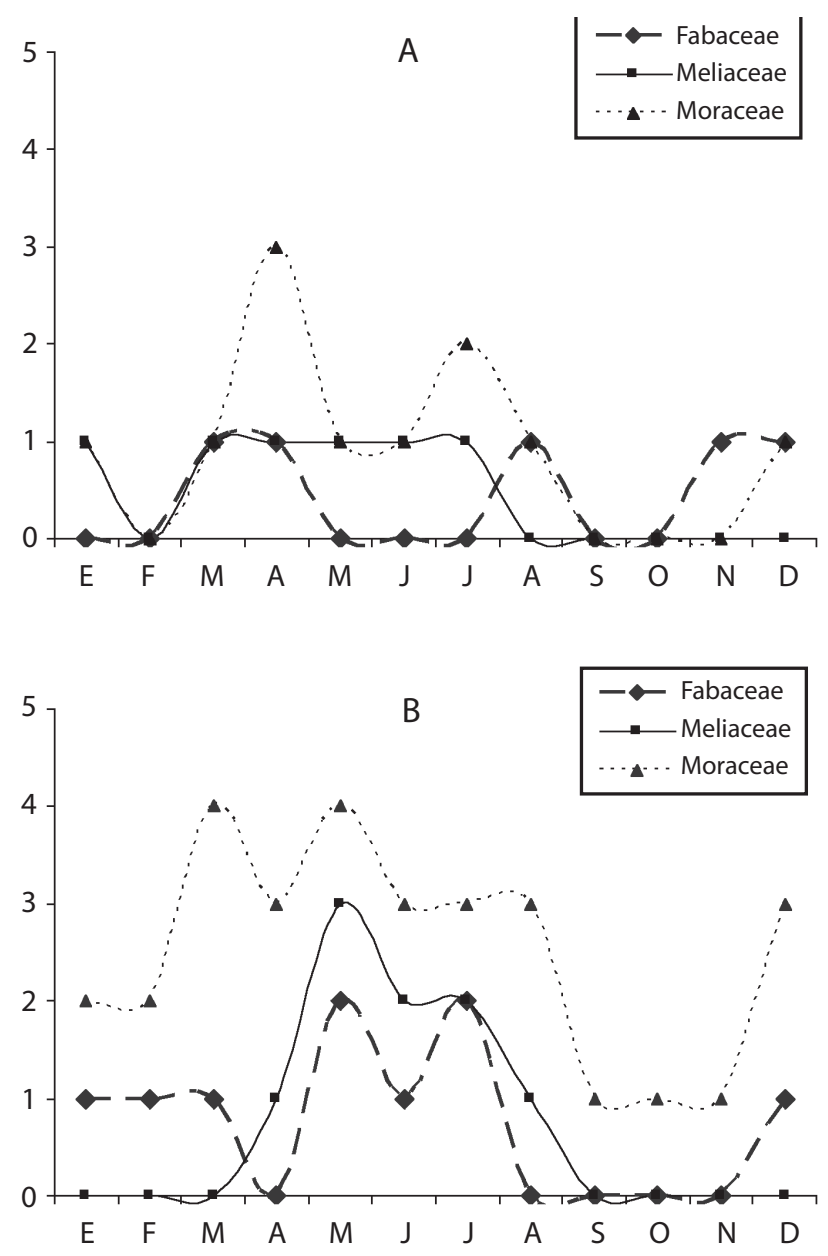

Fig. 3. Fenología de las familias Fabaceae, Meliaceae y Moraceae (A) Floración, (B) Fructificación.

Fig. 3. Phenology of Fabaceae, Meliaceae and Moraceae (A) Flowering, (B) Fruiting.

(2000) en la Selva Lacandona, Chiapas en condiciones tropicales similares. Wright y van Schaik (1994) en un trabajo realizado también con especies arbóreas en Barro Colorado, mencionan que la coincidencia de un periodo de mayor floración en el periodo de mayor sequía se corresponde con una fase de mayor irradiación y de menores niveles de plagas por insectos. Morellato et al. (2000) en un estudio realizado con especies arbóreas en los bosques tropicales del Atlántico del sureste de Brasil, encontraron que el periodo de floración y de producción de hojas nuevas ocurrió poco antes del periodo de mayor humedad, relacionándolo con la longitud del día y temperatura.

De los picos fenológicos encontrados, solo se encuentran diferencias estadísticas entre la floración de marzo-abril, comparada con la de enero-febrero. Con respecto a la fructificación, ésta varió a lo largo del año significativamente, pero los picos de fructificación no fueron significativos con relación a la cantidad de especies fructificando en los otros meses, esto puede deberse a que en la zona de estudio no hay una estacionalidad marcada y por ello el periodo de fructificación de las especies se manifiesta 
a lo largo del año (Sun et al. 1996, Borchert 1998, Morellato et al. 2000). Cabe señalar, que cuando se analizaron los periodos de floración y fructificación de manera separada para cada año correlacionándola con la precipitación mensual del año correspondiente, los patrones difirieron notablemente entre los dos años. Lo mismo ocurrió cuando se compararon los patrones reproductivos a nivel de las dos localidades estudiadas. Sólo se logró encontrar el patrón reportado en este estudio, cuando los datos fenológicos y de precipitación se analizaron integrando los datos de ambos años en ambas localidades, lo cual sugiere que estos estudios deben de abordarse a través de varios años y que un solo periodo no es suficiente para especies arbóreas. En particular en la zona de estudio, durante los años involucrados, el promedio de la precipitación anual fue ligeramente mayor a los $2000 \mathrm{~mm}$, sin embargo, los periodos de sequía y mayor precipitación variaron entre años, mostrando patrones diferentes. Solamente marzo pudo considerarse como mes seco en el 2005, ya que estuvo por debajo de los $50 \mathrm{~mm}$ (que de acuerdo con Vílchez y Rocha (2004) es el valor que se puede tomar como referencia para determinar si un mes es o no es considerado como seco). En el 2004, todos los meses estuvieron por encima de dicho mínimo.

En las montañas de Tenosique no se presenta una estacionalidad marcada que favorezca la pérdida del follaje, por lo que el bosque tropical de esta región, se puede caracterizar como perennifolio. Al respecto Borchert (1998) señala que en la Amazonia, reducciones de la precipitación anual de $>2500 \mathrm{~mm}$ a alrededor de los $1800 \mathrm{~mm}$ no afectaría la fisonomía del bosque. No obstante, algunas especies pueden perder sus hojas totalmente (ej. Cedrela odorata) durante el período de menor precipitación (Marzo-Abril).

No se hicieron análisis de correlación de la fenología de las especies con la temperatura, ya que en las regiones tropicales esta variable es estadísticamente similar a lo largo del año. La disponibilidad de agua a través de la precipitación suele fluctuar en mayor o menor grado siendo ésta la variable de mayor peso a medir en los estudios fenológicos en el bosque tropical (Vílchez et al. 2004).

Cuando se hizo el análisis de la fenología por familia botánica, se encontró que cada una presenta patrones fenológicos particulares, que no necesariamente coinciden con el patrón general observado. Asimismo, se puede observar, que cada especie presenta un patrón propio. Por ello, para comprender la relación de las especies con los factores ambientales que regulan el inicio y el final de sus fases fenológicas, son necesarios estudios mas detallados para especies de interés, tratando de establecer su posible relación con polinizadores $\mathrm{u}$ otra relación interespecífica (Sakai 2001, Vílchez et al. 2004). En este sentido, Plana (2000) señala que algunas especies, especialmente las pioneras, florecen a lo largo del año mientras que las especies de fases sucesionales tardías pueden presentarse en periodos marcados anualmente o incluso cada dos o tres años, tardando periodos de hasta varios años para la primera actividad reproductiva. Aunque en el análisis sólo se incluyen los patrones fenológicos de las familias con mayor número de especies, los datos de floración y fructificación se asientan para cada una de las especies en el Anexo 1.

Como es conocido, en los bosques tropicales coexisten muchas especies de baja abundancia (Colinvaux 1980, Hubbell y Foster 1986), por ello, el conocimiento de los periodos de reproducción de las especies es de gran importancia en estas áreas, ya que muchas de las especies arbóreas no son auto-fértiles (Plana 2000), y debido a la acción antrópica, la abundancia de éstas especies puede ser aun mas limitada, lo que pone en entredicho su supervivencia.

En éste estudio, el $17 \%$ de las especies no mostró alguna fase fenológica reproductiva a lo largo de los dos años evaluados. Esto pudo deberse a que los $10 \mathrm{~cm}$ de dap definidos como tamaño mínimo incluyó árboles sexualmente inmaduros, o a que como se menciono anteriormente, algunas especies tienen periodos reproductivos bi o tri-anuales. Por otra parte se encontró mayor número de especies en fructificación (6\%) que los vistos en floración, 
esto se puede deber a que las observaciones fueron mensuales y se pudo perder la observación de alguna fase corta de floración (Vílchez et al. 2004) o también a que algunas flores son inconspicuas y pasaron desapercibidas al momento de la evaluación. Por ello, sería recomendable realizar las evaluaciones semanal o al menos quincenalmente.

A modo de conclusión podemos decir que los patrones fenológicos de las especies arbóreas varían dentro de una misma especie, entre años y entre localidades (Mantovani et al. 2003). Los datos que se aportan en el presente trabajo también son relevantes para definir los periodos apropiados para la recolecta de semillas y la realización de otras practicas para la obtención de plántulas para el manejo de las especies arbóreas tropicales.

\section{AGRADECIMIENTOS}

Agradecemos a Edgardo E. Fuentes Pech, Marcos Antonio Hernández Vázquez, y Angel Adiel Rodríguez Martínez por su apoyo en la toma de datos de campo. Fondos Mixtos Conacyt-Tabasco dio apoyo financiero mediante el proyecto Manejo de semillas y plántulas de especies arbóreas de las montañas del mpio. de Tenosique, Tabasco: bases para su manejo y conservación TAB-2003-C03-11261. La Comisión Nacional Forestal apoyó al proyecto Estudio de la fenología de especies forestales de interés potencial para el uso económico, ecológico y de conservación. A El Colegio de la Frontera Sur por su apoyo en infraestructura. A los ejidos de Niños Héroes y Nuevo Progreso, y al municipio de Tenosique, quienes amablemente nos permitieron el acceso a sus áreas boscosas y a los guías de campo Isidro Pérez Torres, Mateos José Lucano y Vicente López Moreno.

\section{RESUMEN}

La fenología de 341 árboles que corresponden a 75 especies fue estudiada en las montañas de Tenosique, Tabasco, entre agosto del 2003 hasta agosto del 2005. Para ello, se establecieron cinco transectos de $500 \mathrm{~m}$ de largo por cinco metros de ancho, los cuales se evaluaron mensualmente. Si alguno de los individuos de las especies tenía flores o frutos, la especie como un todo fue considerada en floración o fructificación en ese mes. Se aplicaron pruebas de homogeneidad de ji-cuadrada para evaluar la homogeneidad de la floración y fructificación a lo largo del año y por periodos bimensuales. La floración fue bimodal, con un pico de mayor floración de marzo a abril que corresponde a menor precipitación. En julio se presentó un segundo pico de floración con 21 especies, correspondiendo igualmente a un decremento de la precipitación. El mayor número de especies con fruto se presentó entre mayo y junio, el cual no fue significativo estadísticamente. También se analizan los patrones fenológicos de las familias con mayor número de especies, los cuales difirieron entre sí. No se encontró relación entre el número de especies floreciendo o fructificando y la precipitación promedio mensual. Se encontraron picos fenológicos poco marcados debido a que en la zona de estudio la estacionalidad es poco marcada. Los patrones fenológicos varían dentro de una especie, entre años y entre localidades. Los datos que se aportan en el presente trabajo son relevantes para definir los periodos apropiados de recolecta de semillas para la realización de prácticas de manejo y conservación.

Palabras Clave: fenofases, flora leñosa, bosque tropical, árboles tropicales, biología reproductiva.

\section{REFERENCIAS}

Bencke, C.S.C. \& L.P.C. Morellato. 2002. Comparação de dois métodos de avaliação da fenologia de plantas, sua interpretação e representação. Rev. Bras. Bot. 25: 269-275.

Borchert, R. 1998. Responses of tropical trees to rainfall seasonality and its long term changes. Climatic Change 39: 381-393.

Budowski, G. 1954. La identificación en el campo de los árboles forestales más importantes de la América Central. Instituto Interamericano de Ciencias Agrícolas, Turrialba, Costa Rica.

Cabrera, E., M. Sousa \& O. Téllez. 1982. Imágenes de la flora Quintanarroense. Centro de Investigaciones de Quintana Roo. México D.F., México.

Chuine, I. \& E.G. Beaubien. 2001. Phenology is a major determinant of tree species range. Ecol. Lett. 4: 500-510.

Colinvaux, P.A. 1980. Especies comunes y especies raras, p. 573-597. In P.A. Colinvaux (ed.). Introducción a la Ecología. Limusa, México D.F., México. 
Croat, T.B. 1978. Flora of Barro Colorado Island. University of Stanford, Stanford, California, EEUU.

Finney, D.J. 1980. Statistics for biologists. Chapman and Hall, Nueva York, EEUU.

Fournier, L.A. \& C. Charpantier. 1975. El tamaño de la muestra y la frecuencia de observaciones en el estudio de las características fenológicas de los árboles tropicales. Turrialba 25: 45-48.

Galindo, A.A., S. del C. Ruíz A., C.E. Zenteno R., A. Sol S., C. Bouchot C. \& M. Izquierdo R. 2000. Sustento técnico del documento de propuesta de decreto del área natural protegida denominada "Sierra de Tenosique" UJAT-SEDESPA, Gobierno del estado de Tabasco, Villahermosa, Tabasco, México.

Gentry, J.L. Jr. \& P.C. Standley. 1974. Flora of Guatemala. Fieldiana: Botany 24, Part X, Numbers 1 and 2. Field Museum of Natural History, Chicago, EEUU.

Gobierno del Estado. 1997. Municipio de Tenosique. Secretaria de Desarrollo Social y Protección Ambiental, Gobierno del Estado de Tabasco. Villahermosa, Tabasco, México.

Holdridge, L.R. 1970. Manual dendrológico para 1000 especies arbóreas en la República de Panamá. Inventarización y demostraciones forestales. Informe Técnico No.1. Organización de las Naciones Unidas para la Agricultura y la Alimentación, Panamá, Panamá.

Hubbell, S.P. \& R.B. Foster. 1986. Commonness and rarity in a Neotropical forest: implications for tropical tree conservation, p. 204-231. In M. Soulé (ed.). Conservation biology: the science of scarcity and diversity. Sinauer, Sunderland, Masachusets, EEUU.

INEGI. 2000. Cuaderno estadístico municipal de Tenosique. Gobierno del Estado de Tabasco, Villahermosa, Tabasco, México.

Justiniano, M.J. \& T.S. Fredericksen. 2000. Phenology of tree species in Bolivian dry forests. Biotropica 32 276-281

Longino, J.T. 1986. A negative correlation between growth and rainfall in a tropical liana. Biotropica 18: $195-200$

Mantovani, M., A.R. Ruschel, M. Sedrez dos Reis, A. Puchalski \& R.O. Nodari. 2003. Fenologia reprodutiva de espécies arbóreas em uma formação secundária da floresta Atlântica. Rev. Árvore 27: 451-458.
Morellato, L.P.C., D.C. Talora, A. Takahasi, C.C. Bencke, E.C. Romera \& V.B. Zipparro. 2000. Phenology of Atlantic rain forest trees: a comparative study. Biotropica 32: 811-823

Nash, D.L. \& L.O. Williams. 1976. Flora of Guatemala. Fieldiana: Botany 24, Part XII. Field Museum of Natural History, Chicago, EEUU.

Ochoa-Gaona, S. \& G. Domínguez-Vázquez. 2000. Distribución y fenología de la flora leñosa de Chajul, Selva Lacandona, Chiapas, México. Brenesia 54: $1-24$.

Ochoa-Gaona, S., I. Bratkévich, L. Romero, B.H.J. de Jong \& J.D. Rostrand. 1990. Descripción de las especies. Informe final proyecto etnobotánica de las especies forestales de la IV Región. Universidad Nacional Agraria, Managua, Nicaragua.

Pennington, T.D. \& J. Sarukhán. 2005. Árboles tropicales de México. Universidad Nacional Autónoma de México y Fondo de Cultura Económica, México D.F., México.

Sakai, S. 2001. Phenological diversity in tropical forests. Popul. Ecol. 43: 77-86.

Salas Estrada, J.B. 1993. Árboles de Nicaragua. Instituto Nicaragüense de Recursos Naturales y del Ambiente, Managua, Nicaragua.

Standley, P.C. \& J.A. Steyermark. 1946-1960. Flora of Guatemala. Fieldiana: Botany 24, Parts. I, II, III, IV, V y VI . Field Museum of Natural History, Chicago, EEUU.

Standley, P.C. \& L.O. Williams. 1961-1975. Flora of Guatemala. Fieldiana: Botany 24, Part VII, VIII, IX, XI. Field Museum of Natural History, Chicago, EEUU.

Steyermark, J.A. 1950. Flora of Guatemala. Ecology 31: 368-372.

Sun, C., B.A. Kaplin, K.A. Kristensen, V. Munyaligoga, J. Mvukiyumwami, K.K. Kajondo \& T.C. Moermond. 1996. Tree phenology in a tropical montane forest in Rwanda. Biotropica 28: 668-681.

Talora, D.C. \& P.C. Morellato. 2000. Fenologia de espécies arbóreas em floresta de planície litorânea do sudeste do Brasil. Rev. Bras. Bot. 23: 13-26.

Vílchez, B. \& O. Rocha. 2004. Fenología y biología reproductiva del nazareno (Peltogyne purpurea Pittier) en 
un bosque intervenido de la Península de Osa, Costa Rica, América Central. Kurú: Rev. For. 1: 1-14.

Vílchez, B., R. Chazdon \& A. Redondo. 2004. Fenología reproductiva de cinco especies forestales del bosque secundario tropical. Kurú: Rev. For 1: 1-10.

Wright, S.J. \& C.P. van Schaik. 1994. Light and the phenology of tropical trees. Am. Nat. 143: 192-199.

Zar, J.H. 1999. Biostatistical analysis. Prentice Hall, Nueva Jersey, EEUU.

\section{REFERENCIAS DE INTERNET}

DOF. 1995. Aviso mediante el cual se informa al público en general que están a su disposición los estudios realizados para justificar la expedición del Decreto por el que se pretende declarar como área natural protegida con el carácter de Área de Protección de Flora y Fauna, la zona conocida como Cañón del Usumacinta, localizada en el Municipio de Tenosique, en el Estado de Tabasco. Diario Oficial de la Federación 3 de junio del 2005. $1^{\text {a }}$. Sección. Consultada el 13 de octubre del 2007. (http://www.semarnat.gob.mx/leyesynormas/ La $\% 20$ SEMARNAT\%20en $\% 20$ el $\% 20$ Diario $\% 20$ Oficial $\% 20 \mathrm{de} \% 201 \mathrm{a} \% 20$ Federacin/030605.5.pdf)

ICP Forests. 2006. Forest phenology. International Cooperative Programme on Assessment and
Monitoring of Air Pollution Effects on Forests. Consultada 21 de agosto del 2006. (http://www.metla. fi/eu/icp/phenology/homepage.htm)

Plana, E. 2000. Introducción a la ecología y dinámica del bosque tropical. Curso sobre gestión y conservación de bosques tropicales. Centro Tecnológico Forestal de Cataluña. Consultada el 6 de octubre del 2006. (http://www.ctfc.es/webcast/areas/politica_for/documents/ponb.pdf)

Preuhsler, T., A. Bastrup-Birk \& E. Beuker. 2006. Manual on methods and criteria for harmonized sampling, assessment, monitoring and analysis of the effects of air pollution on forests Part IX Phenological Observations. United Nations Economic Commission for Europe Convention on Long-Range Transboundary Air Pollution. International Cooperative Programme on Assessment and Monitoring of Air Pollution Effects on Forests. Consultada el 22 de agosto del 2006, (http://www.metla.fi/eu/icp/phenology/manualpheno.pdf)

Secretaría de Gobernación. Enciclopedia de los municipios de México, Estado de Tabasco, Tenosique. Instituto Nacional para el Federalismo y el Desarrollo Municipal (INAFED) en coordinación con los Gobiernos de los Estados. Consultada el 6 de septiembre del 2006. (http://www.e-local.gob.mx/wb2/ ELOCAL/EMM_tabasco) 


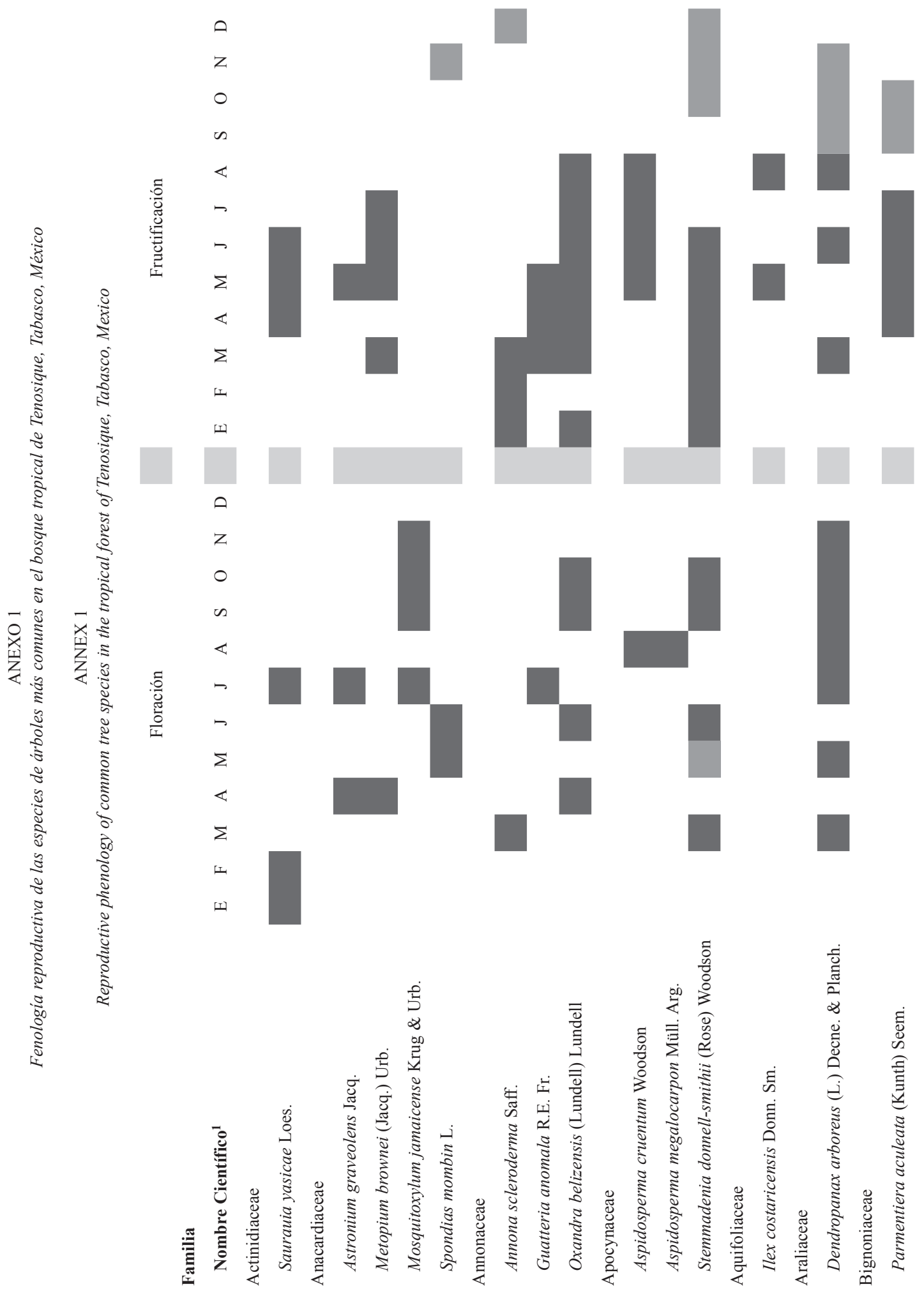




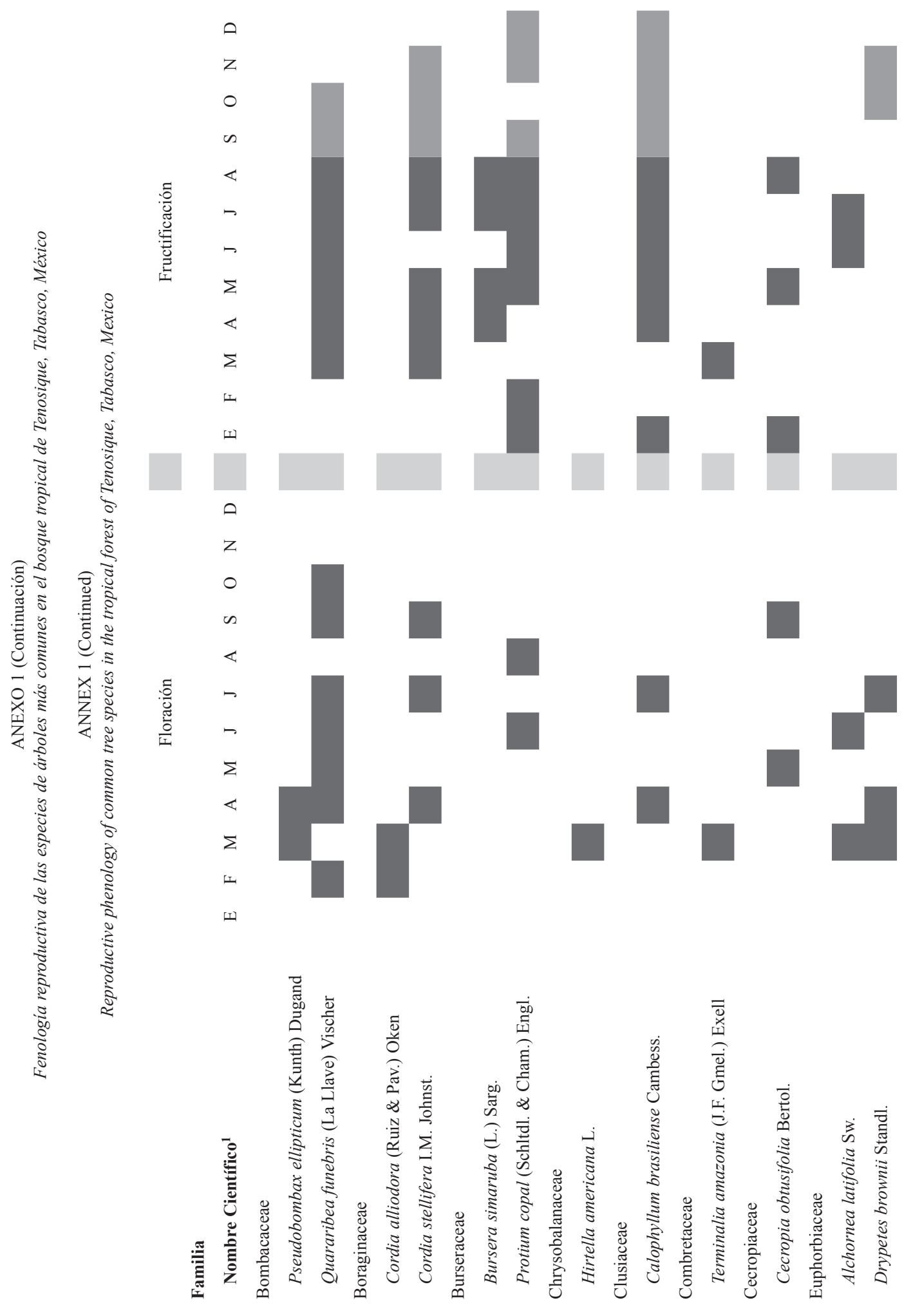




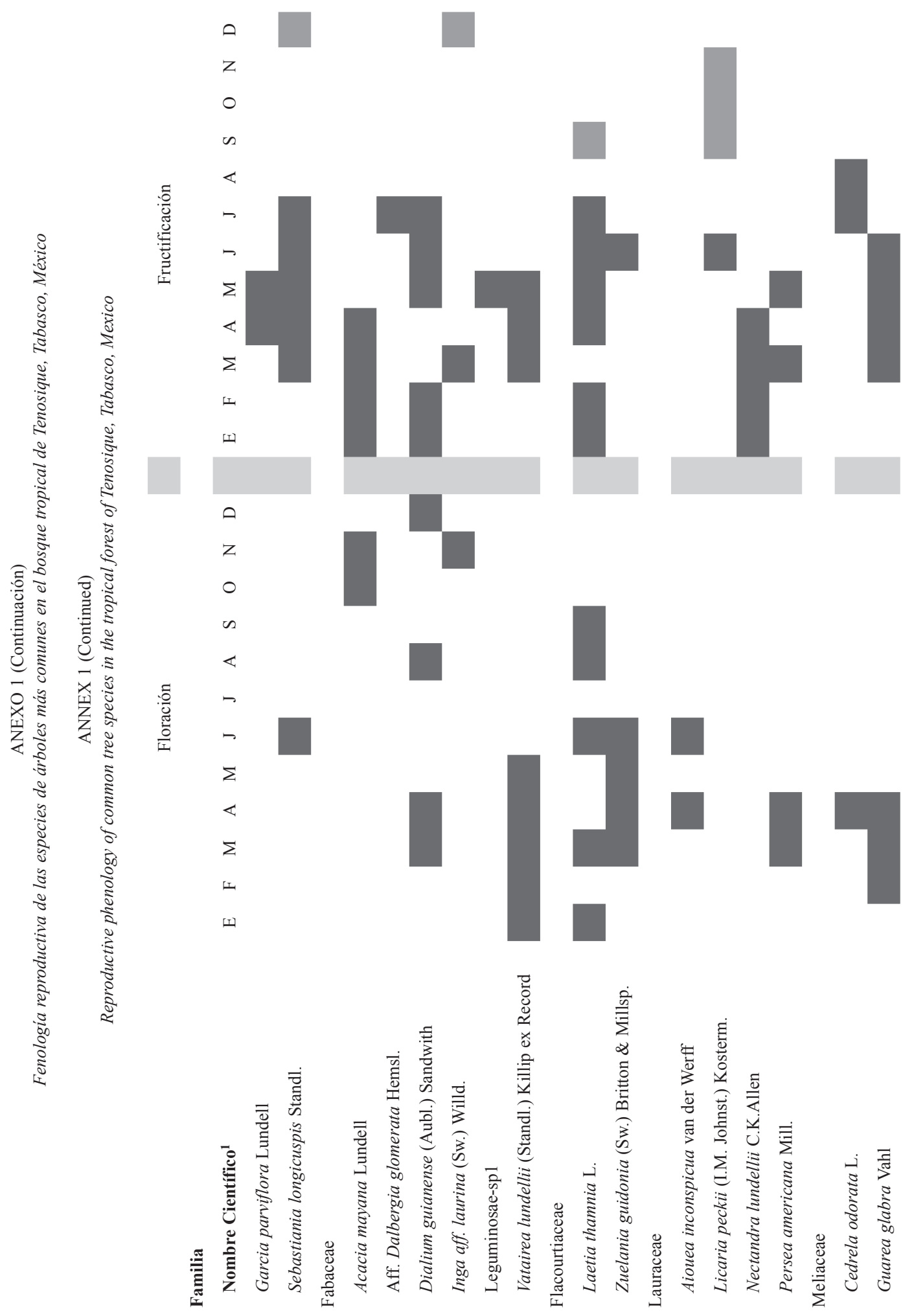




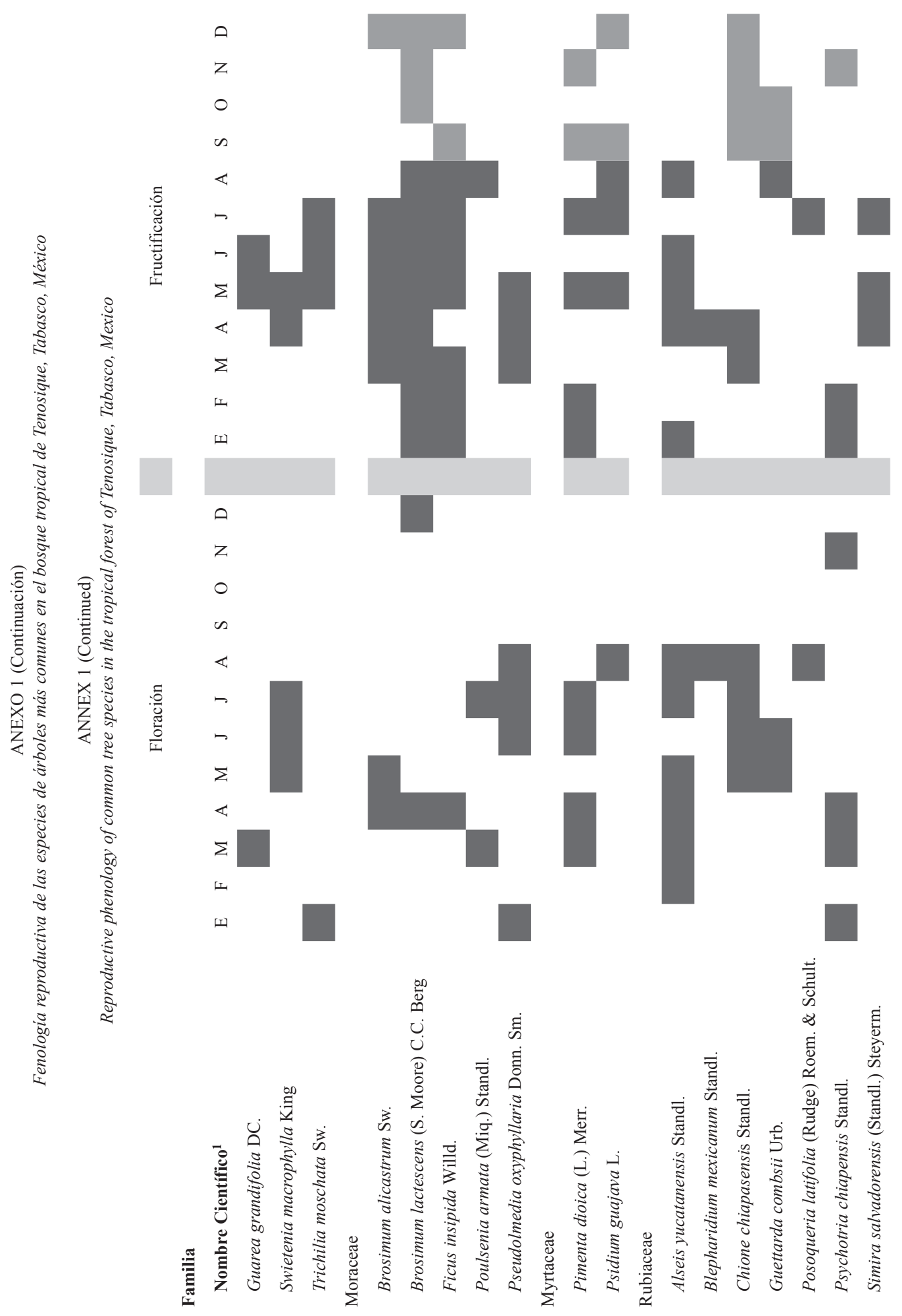




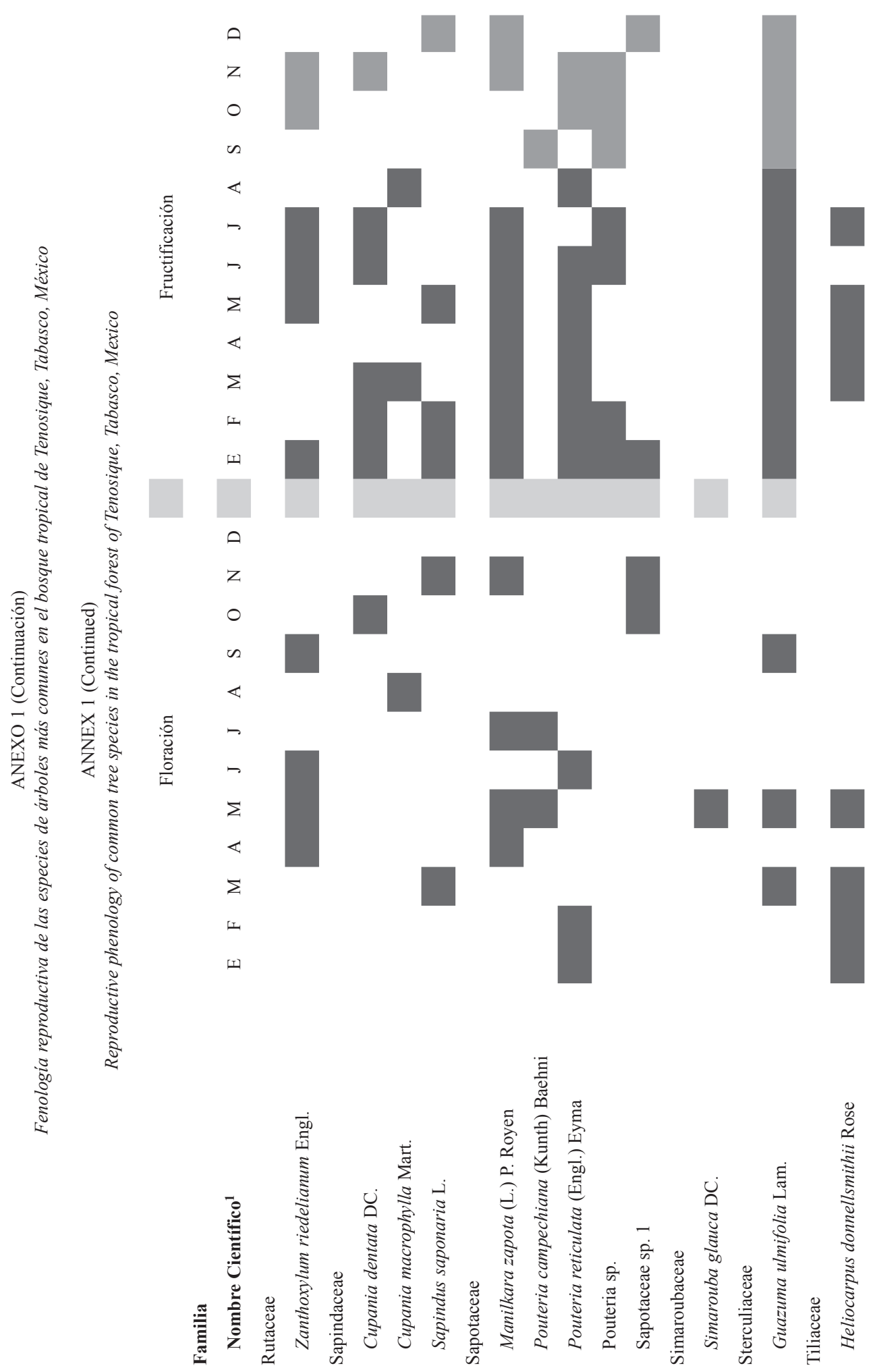




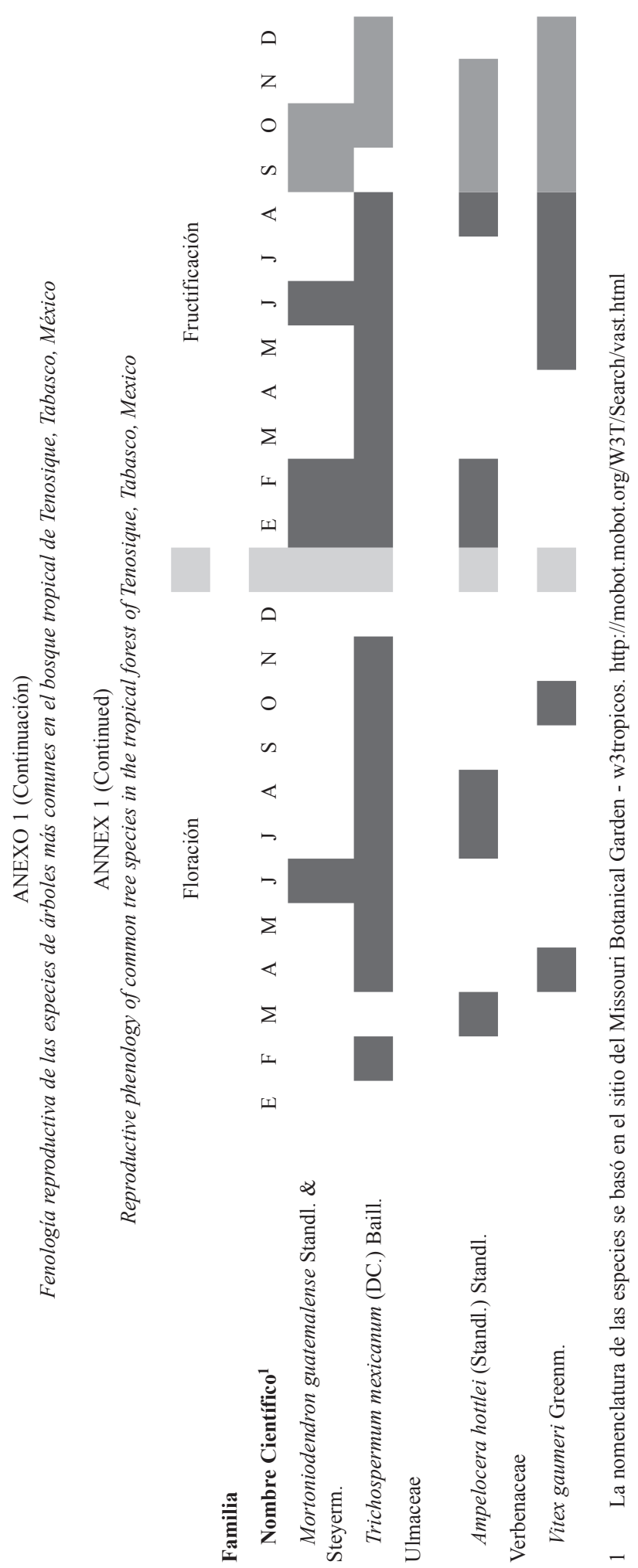

